\section{A Note Concerning a Recent Comparison of Hydrogen-Like and Hartree-Fock Calculations of the Imaginary Parts of Atomic Scattering Factors for Silicon}

\author{
G. Hildebrandt and J. D. Stephenson
}

Fritz-Haber-Institut der Max-Planck-Gesellschaft, Berlin

\section{(Z. Naturforsch. 30 a, 1493-1494 [1975] ; received October 7,1975 )}

A comparison is given between hydrogen-like and Hartree-Fock calculated and experimental absorption data for Silicon. Some values omitted in an earlier paper are added.

1. Recent theoretical determinations of the imaginary parts of the atomic scattering factors of Si for 12 characteristic X-ray wavelengths using both hydrogen-like ${ }^{1}$ and Hartree-Fock eigenfunctions ${ }^{2}$ have shown that both theoretical approaches give almost identical results for this particular element and X-ray energy range. Nevertheless we wish to reemphasize in this note the limitations of the H-like theory and the $\mathrm{X}$-ray energy regions to which it can be applied successfully.

For the more extensive hydrogen-like calculations given in ${ }^{3}$ for elements $Z=6$ to 54 and 18 characteristic wavelengths in the medium $\mathrm{X}$-ray energy range 5 to $25 \mathrm{keV}$ the comparison given in ${ }^{4}$ shows that the hydrogen-like calculations are in satisfactory agreement with other more rigorous theoretical values and those estimable from measured X-ray absorption coefficients only if we consider elements whose values lie between the following limits

$$
6 \lesssim Z \lesssim Z_{E(2 \mathrm{~s})},
$$

where $Z_{E(2 \mathrm{~s})}$ is the atomic number of an element having the $2 \mathrm{~s}$ electron (hydrogenlike) eigenvalue closest to that of the incident X-ray photon energy. This region restricts the calculations to the major $\mathrm{K}$ and $\mathrm{L}$ atomic absorption shells.

Further we show in Table 1, that the H-like calculations given for $\mathrm{Si}$ in both ${ }^{1,2}$ are in slightly better agreement with experiment than the H-F-calculations ${ }^{2}$ when other scattering contributions $\Delta$ (CS, TDS), estimated from the theoretical values given in ${ }^{5}$, are added.

For comparison the values given in the recent edition of the "International Tables for X-Ray Crystallography" 7 are also repeated in Table 1 . These data are mean weighted values obtained from many experimental and theoretical sources. They show remarkable disagreement with the other theoretical and experimental values in Table 1 .

2 . We wish to correct several errors and omissions which have occurred in our earlier published papers.

Table 1. Linear absorption coefficients $\mathrm{cm}^{-1}$ for Si. The mass absorption coefficients $\mu_{0} / \varrho$ given in the "International Tables..." 7 have been converted to linear absorption coefficients using $\varrho S i=2.3283 \mathrm{~g} \cdot \mathrm{cm}^{-3}$.

\begin{tabular}{|c|c|c|c|c|c|c|c|c|}
\hline \multirow[b]{2}{*}{ line } & \multicolumn{2}{|c|}{ Theory } & \multicolumn{4}{|c|}{ Theory, corr. } & \multirow{2}{*}{$\underset{6}{\text { Experiment }}$} & \multirow{2}{*}{$\underset{7}{\text { Int. Tabl. }}$} \\
\hline & $\mathrm{H}-\mathrm{F}^{2}$ & H-like ${ }^{1,3}$ & $\mathbf{\Delta}^{5}$ & $\mathrm{H}-\mathrm{F}$ & H-like & 1 & & \\
\hline $\operatorname{AgK} \alpha$ & 7.28 & 6.94 & 0.34 & 7.62 & 7.28 & 7.32 & 7.61 & 7.58 \\
\hline MoKa & 14.79 & 14.25 & 0.4 & 15.19 & 14.65 & 14.6 & 14.7 & 15.21 \\
\hline $\mathrm{CuK} \alpha$ & 144.3 & 142.9 & 0.8 & 145.1 & 143.7 & 144 & 137 & 152.1 \\
\hline $\mathrm{CoK} \alpha$ & 221.5 & 220.7 & 0.8 & 222.3 & 221.5 & - & 214 & 233.8 \\
\hline $\mathrm{FeK} \alpha$ & 277.2 & 277.2 & 1.0 & 278.2 & 278.2 & 275 & 270 & 293.4 \\
\hline $\mathrm{CrK} \alpha$ & 44.5 .5 & 448.2 & 1.2 & 446.7 & 449.4 & - & 445 & 471.9 \\
\hline
\end{tabular}

Table 2. H-like photoelectric atomic absorption cross sections $(T:=\tau)$, calculated for $\mathrm{Xe}$, together with the value $Q=$ $\tau \mathrm{Q} / \tau$ used in anomalous absorption calculations.

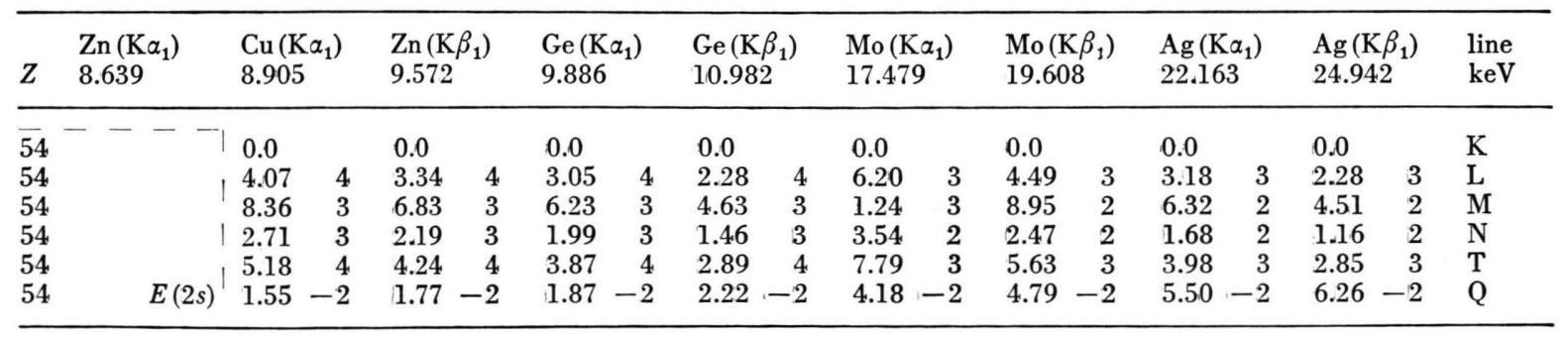

Reprint requests to Prof. Dr. G. Hildebrandt, Fritz-Haber-Institut der Max-Planck-Gesellschaft, D-1000 Berlin 33, Faraday-Weg $4-6$. 
a) Z. Naturforsch. 28 a, 588 (1973) ${ }^{1}$ : In Table 1 the $3 \mathrm{~s}$ screening column should be relabelled $3 \mathrm{~s}, 3 \mathrm{p}$ and the $3 \mathrm{p}, 3 \mathrm{~d}$ column relabelled $3 \mathrm{~d}$ (the correct table of screening constants is given in ${ }^{3}$ ).

b) Z. Naturforsch. 30 a, 697 (1975) ${ }^{3}$ : The H-like photoelectric atomic absorption cross sections for $Z=54$ in barn/atom, unfortunately omitted in Table 3 , are given above.

1 G. Hildebrandt, J. D. Stephenson, and H. Wagenfeld, Z. Naturforsch. 28 a, 588 [1973].

2 M. Calamiotou and S. E. Filippakis, J. Phys. C.; Solid State Phys. 7 (1974).

3 G. Hildebrandt, J. D. Stephenson, and H. Wagenfeld, Z. Naturforsch. 30 a, 697 [1975].

4 J. D. Stephenson, Z. Naturforsch. 30 a [1975], in print.

5 E. Storm and H. J. Israel, Nuclear Data Tables A 7, 565 [1970]. c) Z. Naturforsch. 30 a, 1133 [1975] ${ }^{4}$ : The Hlike photoeffect mass absorption coefficient for $\mathrm{Al}(Z=13)$ using $\mathrm{CoK} \alpha \mathrm{l}$ radiation (Table 3, page 1141) is $73.26 \mathrm{~cm}^{2} / \mathrm{g}$ (and not 92.23 $\left.\mathrm{cm}^{2} / \mathrm{g}\right)$. In the first column of the same table (page 1140) $\mathrm{Ni}$ is misprinted as $\mathrm{Na}$ and in section 3.1.2 (page 1139) Co should replace Zn.

3 . Some remarks concerning the calculation of contributions to anomalous absorption by means of the above data are given elsewhere ${ }^{8}$.

6 J. Stiglich, R. J. Weiss, and A. M. Hansen, in: Adinterim Special Commission on Electron Charge, Spin and Momentum Density, Watertown, Mass., May 1974.

7 International Tables for X-Ray Crystallography, Vol. IV. The Kynoch Press, Birmingham 1974.

8 G. Hildebrandt, J. D. Stephenson, and H. Wagenfeld, Phys. Stat. Sol. (a) 30, K 49 [1975]. 